\title{
Análise dos Modelos de Plataformas Satélites para Distribuição de Cargas em Ambiente Urbano
}

\author{
Analysis of Models of Load Distribution in Urban Environment \\ Análisis de los modelos de plataformas de satélite para la distribución de cargas sobre \\ el medio ambiente urbano
}

Cauana Mendes e Mendes Estudante, Pontifícia Universidade Católica de Campinas, Brasil. cauanamendes@hotmail.com

Marcius Fabius Henriques de Carvalho Professor Doutor, Pontifícia Universidade Católica de Campinas, Brasil. marcius@puc-campinas.edu.br 


\section{RESUMO}

Com o crescente interesse em equilíbrio entre o social, ambiental o econômico, governo e empresas se movimentam no sentido de discutir e implementar ações que contornem os efeitos negativos de atividades no meio urbano. As operações logísticas afetam a qualidade do ar, geram ruídos e vibrações, causam acidentes e contribuem significativamente para a diminuição da qualidade de vida no meio urbano. Ações como, o transporte de carga na cidade, passam a ser regulamentadas por janela de tempo e capacidade de veículo no sentido de contribuir para a melhoria da qualidade de vida dos cidadãos do meio urbano. Entretanto, essas ações dificultam o atendimento às necessidades do cidadão diminuído, quando não adequadamente implementadas, a competitividade dos produtos. Para a adequada distribuição de carga em meio urbano são usados principalmente dois modelos: 0 modelo de Centros de distribuição - plataformas satélites e o modelo de corredores de carga. Este artigo se propõe a identificar e analisar o modelo de plataformas satélites para a distribuição de carga em meio urbano por meio de um levantamento bibliográfico e desenvolvimento de modelos de otimização de fluxo em redes para implementação de um cenário de estudo em ambiente urbano.

PALAVRAS-CHAVE: Logística de Cidades, Modelos de Fluxo em Redes, Programação Linear.

\section{SUMMARY}

With the increase of interest in balance: social, environmental and economic aspects, government and private companies are moving efforts to discuss and implement actions to minimize the negative effects of some activities. Logistical operations in the urban environment affects the quality of air, generates noise and vibration, causes accidents and contributes significantly to the decline in the quality of life in urban areas. Some local actions occur, for example, the transportation of load in urban areas are adjusted using time windows, capability of the vehicle and region of acess. This adjustment contributes to the improvement of quality of life, but in other hand, the satisfaction of customer's needs are reduced. For proper distribution of load in urban environment are mainly used two models: The distribution centers model - satellite platforms and freight corridors model. This article aims to identify and analyze the model of satellite platforms for load distribution in urban areas by a literature review and development of models of flow optimization in networking for the implementation of a study scenario in an urban environment.

KEYWORDS: Cities Logistics, Models of Flow Networks, Linear Programming

\section{RESUMEN}

Con el creciente interés en el equilibrio entre lo social, ambiental y económico, el gobierno y las empresas avanzan estudios para la implementación de acciones que minimicen los efectos negativos de algunas actividades sobre entornos urbanos. Las operaciones logísticas afectan la calidad del aire, generan ruido y vibraciones, causan accidentes y contribuyen significativamente a la disminución de la calidad de vida en las zonas urbanas. Acciones como el transporte de carga en la ciudad, serán reguladas por la ventana de tiempo y la capacidad del vehículo, para contribuir a la mejora de la calidad de vida del entorno urbano. Sin embargo, estas acciones hacen que sea difícil satisfacer las necesidades de los ciudadanos disminuyendo, si no se aplican correctamente, la competitividad de los productos. Para la adecuada distribución de carga en las zonas urbanas se utilizan principalmente dos modelos: el modelo de centros de distribución - plataformas satélites y el modelo de vías de carga. Este artículo tiene como objetivo identificar y analizar el modelo de plataformas de satélite para la distribución de carga en las zonas urbanas por medio de una revisión bibliográfica y desarrollar un modelo de optimización de flujo en redes para la implementación de un escenario de estudio en un entorno urbano.

PALABRAS CLAVE: Logística de las Ciudades, Modelo de flujo de redes, Programación lineal. 


\section{1 - INTRODUÇÃO}

Define-se que o desenvolvimento deve estar associado ao interesse da sociedade, tendo visão do futuro e cuidando dos recursos para evitar o colapso da civilização e a partir do qual propõe-se a busca do o equilíbrio entre desenvolvimento econômico e a sociedade, desenvolvimento econômico e ambiente, e sociedade e ambiente, (Elkington, 1998).

Tendo em vista o crescimento populacional e econômico dos últimos anos acompanhado pela demanda por produtos e serviços, cresce também a importância os processos relacionados à distribuição de cargas em meio urbano, que são a parte do processo da cadeia de suprimento que planeja, implementa e controla o fluxo e armazenamento de mercadorias e serviços, e relaciona informações do ponto de origem ao ponto de destino, (AWASTHI, 2006).

DABLANC, (2007) define a distribuição de carga em meio urbano como um conjunto de fluxos relacionados à entrada, circulação e saída de veículos de transporte de carga em áreas urbanas. A distribuição de carga devem seguir regulamentações de estacionamentos, acesso à via e janela de tempo para as operações de carga/descarga estabelecidas pelos gestores públicos. Dessa forma, as questões de transporte de carga em meio urbano não são consideradas nem quantificadas de maneira sistêmica, não existindo então uma metodologia específica para a sua análise e planejamento.

Com isto há espaço para estudo e aplicação de métodos adequados para este problema afim de minimizar os pontos negativos desta atividade, como por exemplo, o congestionamento, poluição sonora, emissão de poluentes e aumento de acidentes.Segundo Quak (2008), o transporte de cargas em meio urbano gera impactos no chamado "triplo-P" da seguinte forma:

Tabela 1: Impactos do Transporte de Cargas em Meio Urbano no "Triplo-P"

\begin{tabular}{|c|c|c|}
\hline Planeta (Planet) & Pessoas (People) & Economia (Profit) \\
\hline Emissão de poluentes & Problemas respiratórios & Ineficiência e desperdício de recursos \\
\hline Uso de recursos não renováveis & Acidentes de trânsito & Diminuição da acessibilidade urbana \\
\hline $\begin{array}{c}\text { Mudanças climáticas } \\
\text { vibração) }\end{array}$ & $\begin{array}{c}\text { Incomodo (barulho, mau cheiro, } \\
\text { entrega }\end{array}$ & $\begin{array}{c}\text { Diminuição da confiabilidade de } \\
\text { outro) }\end{array}$ \\
\hline $\begin{array}{c}\text { Resíduo de Produtos (pneu, óleo e } \\
\text { Perda e ameaça às espécies } \\
\text { selvagens }\end{array}$ & $\begin{array}{c}\text { Diminuição da atratividade das áreas } \\
\text { centrais }\end{array}$ & $\begin{array}{c}\text { Diminuição do desenvolvimento } \\
\text { econômico }\end{array}$ \\
\hline
\end{tabular}

FONTE: Adaptado de Quak, 2008

A Logística é o processo de planejamento, implementação e controle do fluxo eficiente e economicamente eficaz de matérias-primas, estoque em processo, produtos acabados e informações relacionadas desde o ponto de origemao ponto de consumo visando atender os requisitos do cliente, (BALLOU, 2001). A logística na cidade, tradução livre do termo citylogistics, pode ser considerada um processo de otimização das atividade logística e de transporte desempenhada por empresas privadas, buscando solução econômica considerando 
a infraestrutura urbana disponível, o ambiente de tráfico, o congestionamento e o consumo energético. (TANIGUICHI apud AWASTHI, 2006). Tem como principais agentes:

- Embarcadores, que englobam fabricantes, atacadistas e varejistas;

- Transportadoras de carga e empresas de armazenagem;

- Residentes, que são os consumidores;

- Administradores, que representam o governo ou autoridades de transporte, em nível nacional, estadual e municipal.

Figura 1: Relação entre os agentes da logística

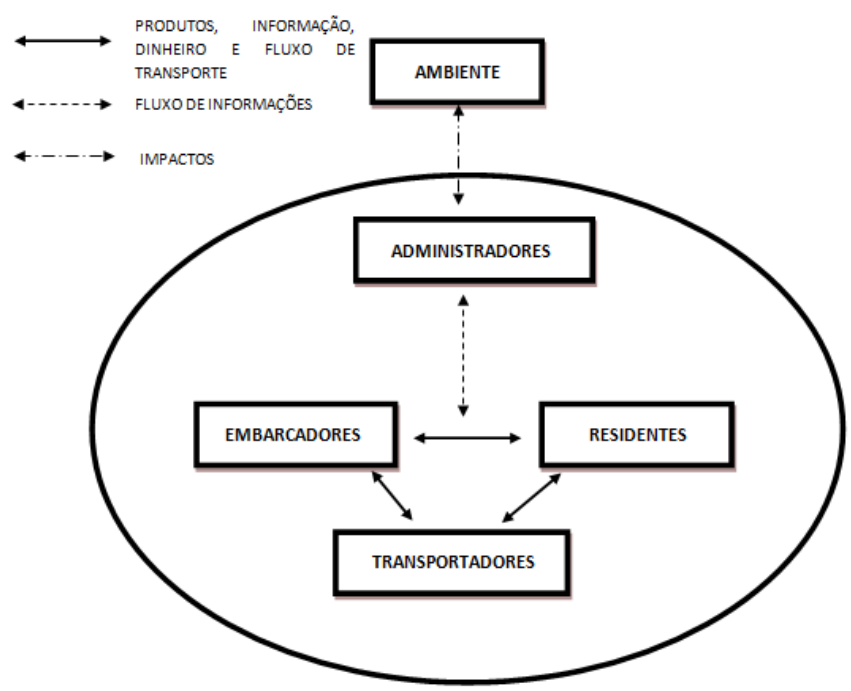

FONTE: AWASTHI, 2006

Além desses fatores, esse trabalho é tambémmotivado pelo entendimento da necessidade de adaptar às regulações impostas ao transporte de cargas em meio urbano, atendendo os clientes de forma mais eficaz e maximizando retorno à empresa.

Uma proposta para solução deste problema é a utilização de uma estrutura em níveis como apresentado na Figura 2, onde aparecem os conceitos de Plataformas Intermodais (PI), Centro de Consolidação de Carga (CCC), e Plataformas Satélites (PS), (BENJELLOUN e CRAINIC, 2009).

Figura 2: Estrutura da rede para suprimento urbano

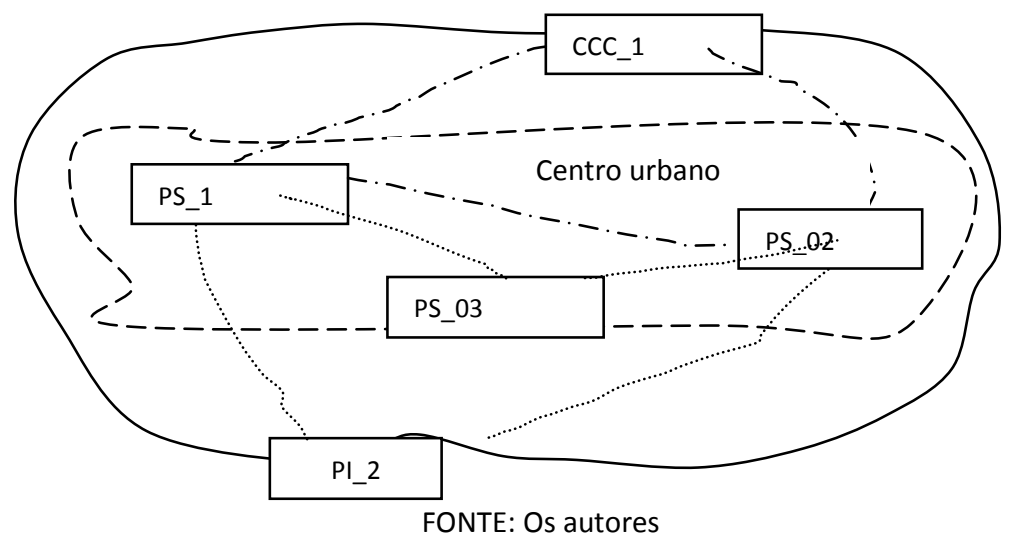


As Plataformas Intermodais (PI) são instalações situadas próximas ao acesso ou ao anel de auto-estrada ou parte de um terminal aéreo, trem ou de navegação de um grande centro urbano e se constitui no primeiro nível. Já o CCC, nível dois, pode ser visto como plataformas intermodais com funcionalidade melhorada para fornecer movimentos de carga eficientes coordenados dentro da zona urbana.Por esta proposta, as atividades de consolidação acontecem nos chamados Centro de Consolidação de Carga (CCC) onde cargas (farmacêuticas, eletrônicos, cosméticos, alimentos etc.) são recebidas de veículos maiores classificadas e consolidadas em veículos menores dedicados às Plataformas Satélites (PS) ou a distribuição em áreas menos densas.

A configuração espacial e a densidade populacional influenciam na determinação do número de CCCs e no número de níveis em que o sistema é projetado (BENJELLOUN e CRAINIC, 2009). Por exemplo, em um primeiro nível podeter-se um CCC localizado na periferia da zona urbana e em um segundo nível construídas PSs que recebem cargas do CCC e de outros pontos. As PSs, nível três, são pontos situados em praças, estacionamentos ou mesmo garagens municipais, onde é feita a transferência de cargas para veículos apropriados para distribuição em centros urbanos densos, tendo como principal característica terem pequena capacidade e estarem adaptados para circular em ruas estreitas. Como estes pontos estão situados no centro urbano, os veículos não podem permanecer por longo tempo parados, requerem operações de transferências rápidas e se destinam a viagens curtas. Em algumas cidades da França estes veículos são elétricos, o que proporciona uma redução significativa de gazes poluentes.

Nas PSs, as cargas são transferidas e consolidadas em um veículo adaptado para distribuição de cargas em zonas densas. Dois tipos de veículos são necessários para a implementação deste sistema: o Caminhão Urbano para movimento da carga até os pontos satélites utilizando rotas selecionadas, projetado no sentido de minimizar o impacto de tráfego e ambiental, e podendo visitar mais de uma plataforma satélite durante sua viagem. Estas viagens (rotas e partidas) podem ser otimizadas e coordenadas com as operações das plataformas satélites inclusive considerando a disponibilidade dos veículos de carga urbana e horários de distribuição. 0 segundo tipo, veículos para a entrega final, pode ser constituído de veículos menores, elétricos, a gás ou bicicleta (MACIEL, 2013). Em resumo, a distribuição de cargas ocorre em níveis, Figura 3. Primeiro o CCC recebe a carga de transportes de longa distância ou das plataformas intermodais, consolida e, de forma coordenada, o distribui para as PSs. O segundo nível (PSs) faz a distribuição dentro de uma área especificada muitas vezes para atendimento de uma característica de carga local. 
Figura 3: Plataforma de Distribuição de Carga Urbana

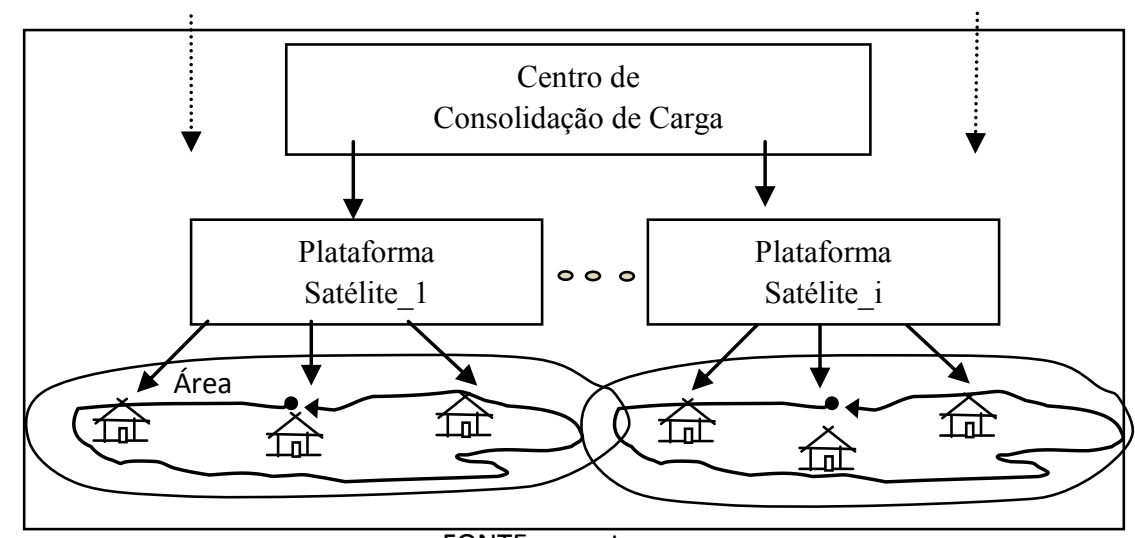

FONTE: os autores

\section{2- OBJETIVO}

Este projeto tem como objetivo a proposição de uma metodologia em dois níveis para representação do sistema de fluxo de abastecimento de um centro urbano. $O$ primeiroconsidera o carregamento de um veículo em um centro de distribuição de carga e de sua rota até a plataforma satélite e a segunda etapa o estabelecimento da melhor rota a partir da plataforma satélite para a distribuição local.

\section{3 - REFERENCIAL TEÓRICO}

Há diversas variáveis que interferem no sistema logístico, sendo exemplo de variáveis externas: economia, política, infraestrutura do transporte, característica socio-culturais e meio ambiente. Além dessas variáveis externas, variáveis internas também interferem no sistema, e são exemplo delas: stakeholders, carga, informação, disponibilidade financeira e eficiência do transporte (AWASTHI, 2006).

A logística nas cidades enfrenta diversos problemas, entre eles (AWASTHI, 2006):

1) Alocação de produtos/bens no veículo;

2) Escolha da melhor rota de entrega;

3) Impacto social e ambiental do transporte de carga em meio urbano. 
Figura 4: Fluxo logístico nas cidades

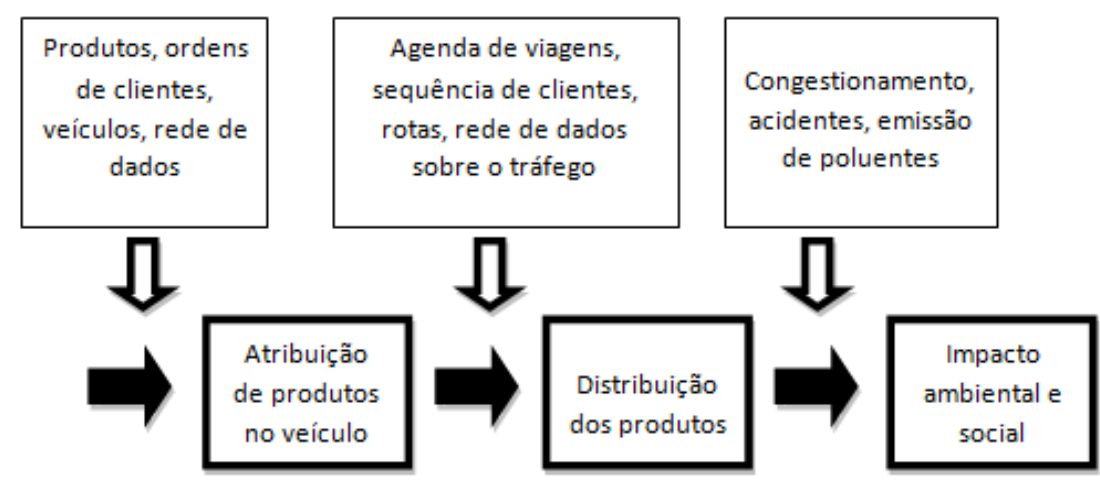

FONTE: Adaptado de Awasthi, 2006

Há dois enfoques principais para a análise de transporte de carga em meio urbano, o primeiro utiliza modelos de otimização e o segundo modelos de simulação. Segundo Moore e Weatherford (2006) a diferença fundamental está no papel das variáveis de decisão dos dois métodos.

- No modelo de otimização, os valores das variáveis de decisão são as saídas. Ou seja, o modelo fornece um conjunto de valores para as variáveis de decisão que otimiza (maximiza / minimiza) o valor da função objetivo.

- No modelo de simulação os valores das variáveis de decisão são entradas. O modelo avalia a função objetivo para um determinado conjunto de valores.

Este trabalho está interessado em modelos de fluxo em redes para representação do sistema de fluxo de abastecimento de um centro urbano. Redes são diagramas compostos por uma coleção de vértices ou nós (esquinas, pontos de decisão) ligados entre si por um conjunto de $\operatorname{arcos}$ (caminhos/rotas). Estes modelos são casos especiais de modelos de programação linear e apresentam uma boa visibilidade gráfica. A cada nó está associado um conjunto de opções e cada arco tem associado um custo e o objetivo do problema de otimização é determinar o menor custo (tempo, distância) de atendimento à demanda a partir de pontos de suprimento. A estes arcos pode-se também associar capacidades que representariam o fluxo máximo de transporte (veículos) por unidade de tempo permitido em um corredor. No caso do problema de pesquisa há a necessidade de um modelo multifluxo para representar os diversos modais de transporte em análise. Os tempos de carga e descarga e distâncias percorridas por um veículo considerados nesta pesquisa são constantes. Nesta situação os modelos estruturados de otimização, que consideram os parâmetros do modelo perfeitamente conhecidos e constantes, são os mais indicados em relação aos modelos de simulação. Os principais passos para desenvolvimento e experimentação de um modelo otimização para a distribuição de carga pode ser decomposto em dois subproblemas: 


\subsection{ATRIBUIÇÃO DE PRODUTOS AO VEÍCULO}

Uma boa atribuição de produtos em um veículo tem como objetivo minimizar o número de viagens e de veículos utilizados levando em considerações as limitações de capacidade dos veículos e também a natureza da carga, que pode demandar maior cuidado.Uma alternativa para essa otimização é a utilização do Solver (ferramenta computacional do excel), aplicando o método de solução para o problema da mochila que busca determinar, dentre $\mathrm{n}$ possíveis objetos que têm associados um valor de utilidade e um peso, quais os que devem ser carregados em uma mochila. O Objetivo é maximizar o valor da utilidade na mochila contato que não ultrapasse sua capacidade (peso), como apresentado na eq.1.

$\mathrm{Fo}=\operatorname{Max} \sum_{i=1, n}$ cixi (equação 1)

Restrição $\quad \sum_{i=1, n}$ pixi $\leq C \max$

Onde:cj: Utilidade do Objeto

pi: peso do objeto

Cmax: capacidade máxima da mochila

Nesse caso, para maximizar a utilidade da carga na mochila sem ultrapassar a capacidade máxima, segundo o Solver, o ideal é que seja colocado: a barra de cereal, o casaco, o celular, a água, o protetor solar, o protetor labial, a garrafa de oxigênio, a câmera fotográfica e a foto do padre.Esse problema simplifica a mesma idéia da alocação de produtos em um caminhão, que devem ser alocados seguindo algum critério de prioridade, como valor monetário, prioridade, utilidade ou urgência, por exemplo, respeitando a capacidade máxima do veículo transportador, que pode ser dada em peso ou volume, por exemplo.

Tabela 2:Utilidade e peso dos itens do problema da mochila

\begin{tabular}{|c|c|c|c|}
\hline Objeto & Legenda & Utilidade & Peso \\
\hline Barra de cereal & X1 & 6 & 200 \\
\hline Casaco & X2 & 7 & 400 \\
\hline Tênis & X3 & 3 & 400 \\
\hline Celular & X4 & 2 & 100 \\
\hline Água & X5 & 9 & 1000 \\
\hline Protetor solar & X6 & 5 & 200 \\
\hline Protetor Labial & X7 & 2 & 30 \\
\hline Garrafa de oxigênio & X8 & 10 & 3000 \\
\hline Máquina fotográfica & X9 & 6 & 50 \\
\hline Foto do Padre & X10 & 1 & 1 \\
\hline
\end{tabular}

FONTE: BELFIORI, et al

Nesse caso, para maximizar a utilidade da carga na mochila sem ultrapassar a capacidade máxima da mesma, segundo o Solver, o ideal é que seja colocado nela: a barra de cereal, o casaco, o celular, a água, o protetor solar, o protetor labial, a garrafa de oxigênio, a câmera fotográfica e a foto do padre. 
Esse problema simplifica a mesma ideia da alocação de produtos em um caminhão, que devem ser alocados seguindo algum critério de prioridade, como valor monetário, prioridade, utilidade ou urgência, por exemplo, respeitando a capacidade máxima do veículo transportador, que pode ser dada em peso ou volume, por exemplo.

\subsection{DISTRIBUIÇÃO DA CARGA}

Outro ponto relevante na logística em centros urbanos é a distribuição de carga, ou seja, a escolha da melhor rota para suprimento da demanda a ser seguida pelos veículos de transporte de modo a minimizar custos percorridas. Uma alternativa para essa escolha éo problema do caixeiro viajanteimplementado em Solver.O problema do caixeiro viajante, supõe a necessidade de se passar por $\mathrm{n}$ pontos ou cidades, de modo a diminuir a distância, custo ou qualquer outra variável de decisão, passando uma vez só por cada ponto.

Esse modelo permite a visualização e entendimento simplicado de um problema de transporte, e exemplifica problemas encontrados para a definição de rotas em um centro urbano para um caminhão, por exemplo. A tabela 3, apresenta as coordenadas das cidades no plano cartesiano para o exemplo em estudo.

Tabela 3: Coordenadas das cidades no plano cartesiano.

\begin{tabular}{|c|c|c|}
\hline Cidade & $\mathbf{X}$ & $\mathbf{Y}$ \\
\hline 1 & 10 & 30 \\
\hline 2 & 20 & 50 \\
\hline 3 & 50 & 90 \\
\hline 4 & 70 & 30 \\
\hline 5 & 90 & 50 \\
\hline
\end{tabular}

FONTE: BELFIORI, et al

A partir da Tabela 3 pode-se estabelecer a distância entre as cidades como na tabela 4.

Tabela 4:Distâncias entre as cidades

\begin{tabular}{|c|c|c|c|c|c|}
\hline & $\mathbf{1}$ & $\mathbf{2}$ & $\mathbf{3}$ & $\mathbf{4}$ & $\mathbf{5}$ \\
\hline $\mathbf{1}$ & 0 & 22,36 & 72,11 & 60 & 82,46 \\
\hline $\mathbf{2}$ & 22,36 & 0 & 50 & 53,85 & 70 \\
\hline $\mathbf{3}$ & 72,11 & 50 & 0 & 63,25 & 56,57 \\
\hline $\mathbf{4}$ & 60 & 53,85 & 63,25 & 0 & 28,28 \\
\hline $\mathbf{5}$ & 82,46 & 70 & 56,57 & 28,28 & 0 \\
\hline
\end{tabular}


Figura 5: Localização das cidades no plano cartesiano

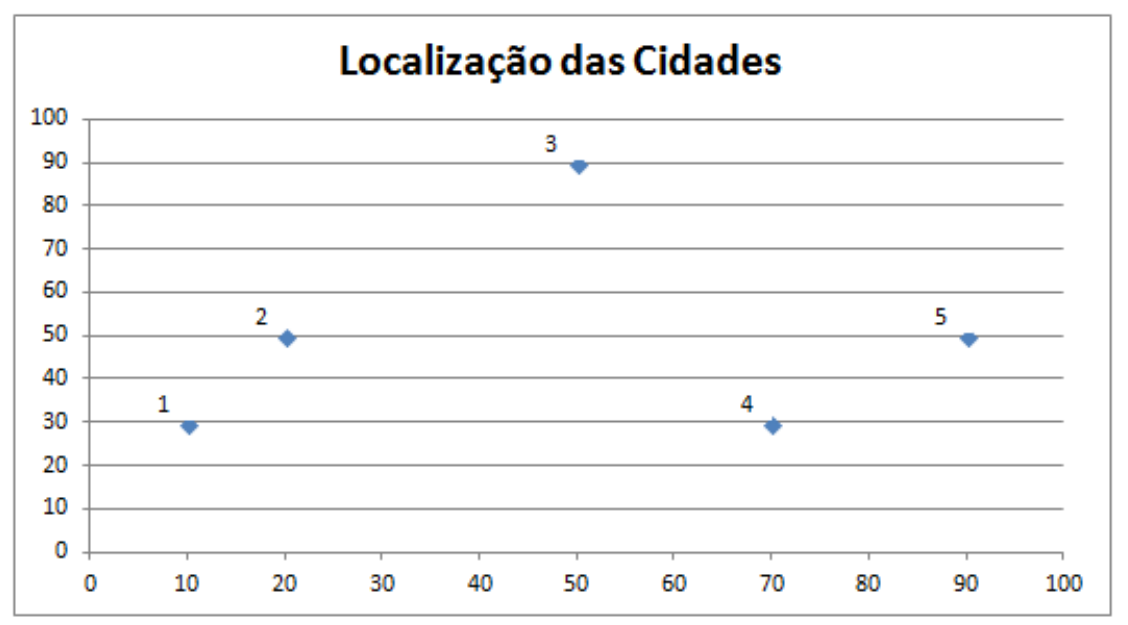

Respeitando todas as restrições do sistema, que garantem que haverá chegada, passagem e saída de cada nó apenas uma vez, e que não se passará pelo menos nó fazendo um loop de $3 \mathrm{e}$ 4 pontos, a solução ótima encontrada pelo Solver tem função objetivo é minimizada para 217,21 unidades de distância e a melhor rota pode ser vista graficamente na figura abaixo:

Figura 6: Rota ótima encontrada pelo Solver

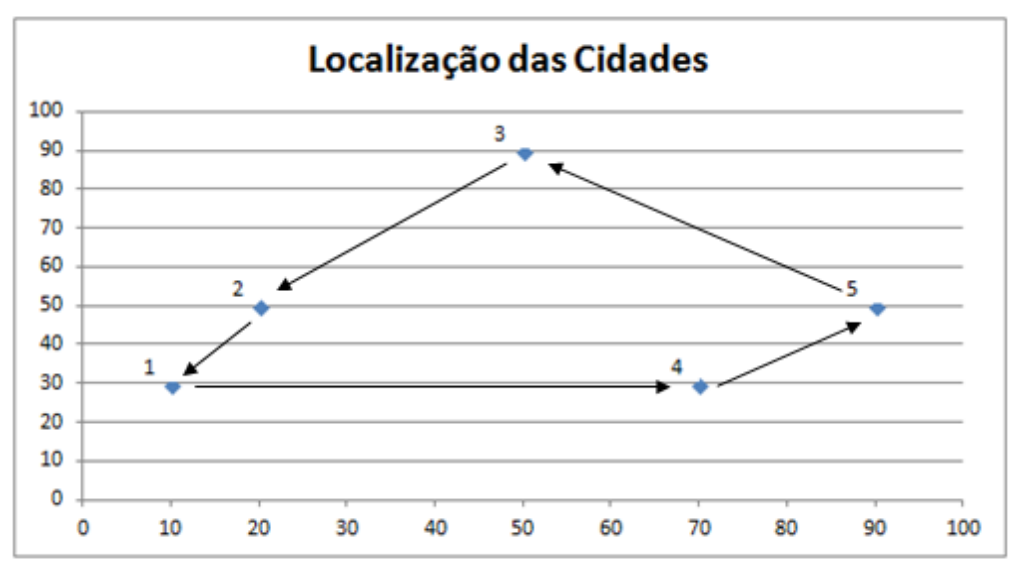

\subsection{IMPACTO SOCIAL E AMBIENTAL DO TRANSPORTE DE CARGA EM MEIO URBANO}

O crescimento populacional acentuado nos centros urbanos ao passar dos anos teve reflexos significativos na demanda de recursos energéticos. Entre eles se destacam os combustíveis como forma de viabilizar a mobilidade urbana no sentido de atender uma população mais distante dos centros de trabalho moradia e lazer. Com isto o número de veículos que cresceu proporcionalmente ao número de pessoas proporcionou um cenário economicamente favorável, mas conduziu ao aumento do uso de combustíveis com consequente elevação da emissão de poluentes na atmosfera, tais como: monóxido de carbono, hidrocarbonetos, óxidos de nitrogênio, óxidos de enxofre e dióxido de carbono, que além de gerarem impactos na 
camada de ozônio favorecendo o efeito estufa e as mudanças climáticas, são nocivos à saúde da população (CARVALHO,2011).

Quantificar as emissões de poluentes é importante para a proposição de medidas mitigadoras de seus efeitos. Uma vez que o papel do planejamento urbano está ligado ao gerenciamento da organização espacial das cidades no intuito de promover o eficiente uso dos serviços e infraestrutura urbana, o dimensionamento da emissão de poluentes atmosféricos permite propor ações para mitigação dos efeitos negativos do transporte de carga contribuindo para um melhor planejamento tanto de novos empreendimentos da cidade, quanto na melhoria dos já existentes, visando a melhoria da qualidade do ar e consequentemente da qualidade de vida da população.

Os transportes de carga são basicamente abastecidos com diesel. Além do diesel, uma alternativa para ser usada, é o biodiesel, um combustível biodegradável produzido a partir de fontes renováveis que podem ser gorduras animais ou óleos vegetais, por exemplo, que podem substituir parcialmente ou totalmente o óleo diesel de petróleo, de forma que o diesel com $2 \%$ de biodiesel é chamado de B2 e assim segue sucessivamente até o B100 que é o biodiesel puro.O $\mathrm{CO} 2$ foi poluente escolhido para abordagem mais abrangente no projeto, ou seja, o cálculo de sua emissão será a base das comparações dentro dos cenários.

Tabela 5: Rendimento quilométrico e emissões de $\mathrm{CO} 2$ por combustível

\begin{tabular}{|c|c|c|c|}
\hline Combustíveis & $\begin{array}{c}\text { Rendimento } \\
\text { energético } \mathbf{~ k m / I}\end{array}$ & $\begin{array}{c}\text { Emissões por fonte } \\
\text { energética kg de CO2// }\end{array}$ & $\begin{array}{c}\text { Emissões por fonte } \\
\text { energética kg de CO2/km }\end{array}$ \\
\hline Gasolina & 10 & 2,8 & 0,28 \\
\hline Álcool & 7 & 0,56 & 0,08 \\
\hline Diesel & 2,5 & 3,2 & 1,28 \\
\hline Gasolina C (25\% de álcool) & 9,25 & 2,24 & 0,24 \\
\hline
\end{tabular}

FONTE: Adaptada de CARVALHO, 2011.

Essa substituição torna possível a redução da emissão de diversos gases, como o CO2, como pode ser visto na tabela abaixo (NOGUEIRA, J., DARBELLO, M.,2009):

Tabela 6: Emissões de poluentes da mistura de diesel e biodiesel

\begin{tabular}{|c|c|c|c|c|c|}
\hline Poluente & Redução/Aumento & B100 (\%) & B20 (\%) & B10 (\%) & B5 (\%) \\
\hline $\begin{array}{c}\text { Gases de efeito } \\
\text { estufa (CO2) }\end{array}$ & $\mathrm{R}$ & 78 & 15 & 7,5 & 3,75 \\
\hline Enxofre & $\mathrm{R}$ & 98 & 19 & 9,5 & 4,95 \\
\hline Material particulado & $\mathrm{R}$ & 50 & 10 & 5 & 2,5 \\
\hline
\end{tabular}

FONTE: NOGUEIRA, J., DARBELLO, M.,2009.

Adicionar até $20 \%$ de biodiesel ao diesel não o desqualifica perante a Portaria no310 da ANP, que estabelece as especificações necessárias para a comercialização de óleo diesel no país, pois tal mistura não modifica drasticamente as propriedades do óleo (Revista Biotecnologia Ciência \&Desenvolvimento,2003). Por isso, para esse projeto, será analisada a utilização do biodiesel B20, que já pode ser incluída em um cenário atual e o biodiesel B100, numa demonstração de uma opção, até então, utópica. Sendo as fórmulas as seguintes: 
- Para o diesel, por unidade de viagem:Empu $=$ km_perc *em_comb_km (equação 2)

- Para o biodiesel B20, por unidade de viagem:Empu $=($ km_perc $*$ em_comb_km) $* 0,85$ (equação 3)

- Para o biodiesel B100, por unidade de viagem:Empu $=\left(\mathrm{km} \_\right.$perc $*$ em_comb_km) $* 0,22$ (equação 4)

Sendo:

Empu:emissão de $\mathrm{CO} 2 \mathrm{em} \mathrm{kg}$ no cenário em estudo por veículos pesados, considerando o número de viagens semanais o número de unidades de ônibus.

Empc:emissão de $\mathrm{CO} 2 \mathrm{em} \mathrm{kg}$ no cenário em estudo por veículos pesados, considerando o número de entradas no dia de maior/menos movimento o número de unidades de caminhões. $\mathrm{km} \_$perc : quilometros percorridos no trajeto.

em_comb_km: emissão de CO2 por quilometro percorrido

\section{ESCOLHA DE UM CENÁRIO EXPERIMENTAL PARA ESTUDO}

\subsection{DIVISÃO EM ÁREAS URBANAS}

Será utilizado como cenário experimental de estudo a região metropolitana de Campinas e o Aeroporto Viracopos, considerado como o Centro de distribuição de carga, é ponto central para se estabelecer uma distribuição de carga até as plataformas satélites, pontos de recebimento de carga, localizadas nas cinco macrozonas de Campinas, segundo a figura 7, com veículos grandes de transporte. Após essa primeira distribuição, pretende-se estabelecer uma segunda distribuição tendo a plataforma satélite de cada área como origem e pontos aleatórios na área como destino, com veículos menores de transporte, adotando sempre valores estimados e aleatórios para simulação de cenários. 
Figura 7: Campinas em cinco áreas

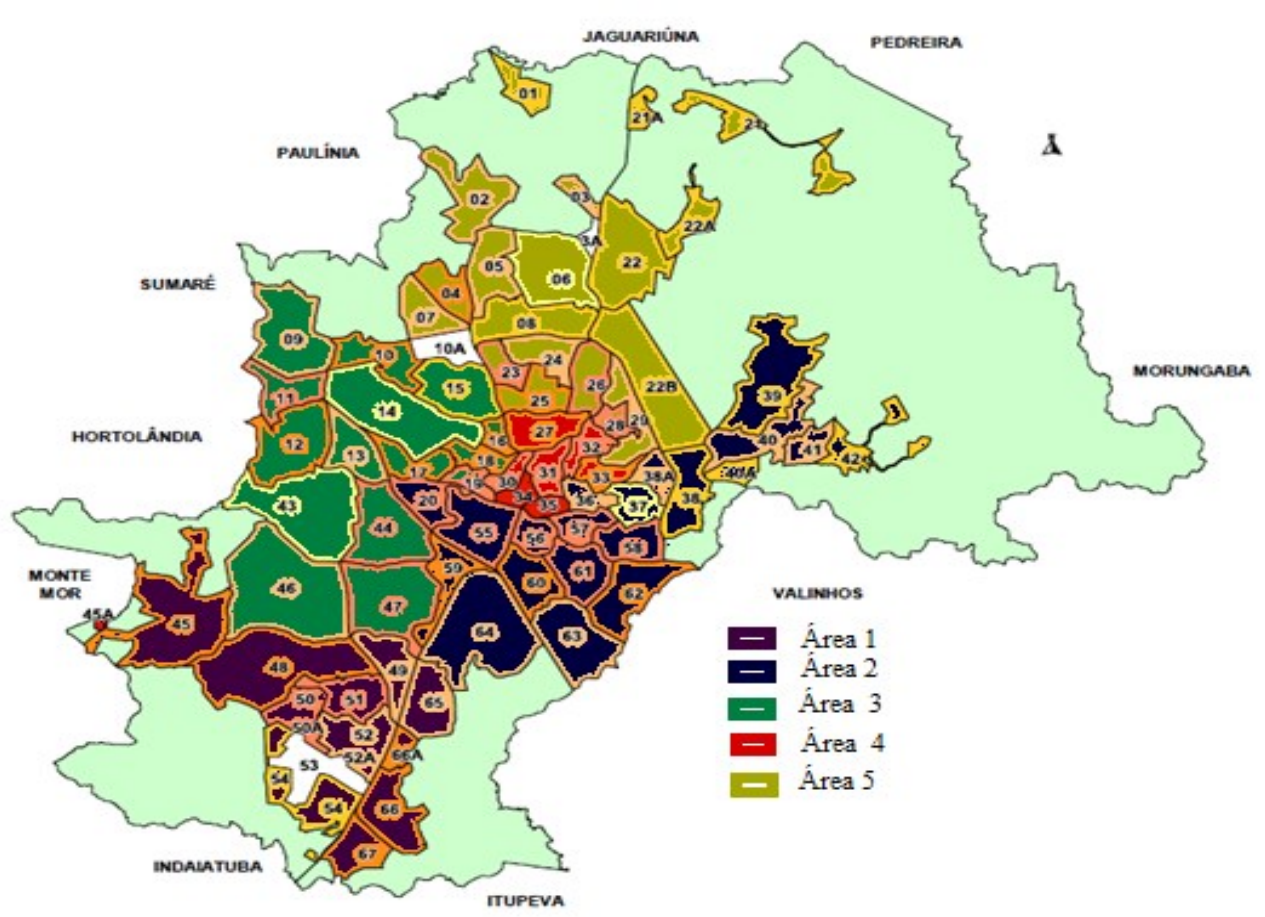

FONTE: (Plano Diretor de Campinas, 2010)

O mapa da figura 7, é dividido de forma a deixar cada área com uma densidade populacional similar às outras, como segue a tabela abaixo, (plano diretor de campinas 2010)

Tabela 7: Características das cinco áreas

\begin{tabular}{|c|c|c|c|}
\hline Área & População Residente & $\begin{array}{c}\text { Renda } \\
\text { Média }\end{array}$ & $\begin{array}{c}\text { Densidade } \\
\text { Populacional }\end{array}$ \\
\hline 1 & 241.717 & 38,87 & 51837,38 \\
\hline 2 & 286.107 & 1708,44 & 62295,22 \\
\hline 3 & 293.093 & 84,85 & 41146,02 \\
\hline 4 & 106.309 & 86,43 & 54826,48 \\
\hline 5 & 131.192 & 132,46 & $37.274,40$ \\
\hline
\end{tabular}

FONTE: (Plano Diretor de Campinas, 2010)

Devido à dificuldade de se obter dados reais relativos à demanda diária e espécie de mercadorias em cada área, além da localidade de cada demanda, o estudo foi realizado com base em estimativas de distância, tempo, demanda, localidade e espécie de mercadoria.

\subsection{SELEÇÃO DE UMA ÁREA PARA APLICAÇÃO DO MODELO}

Para aplicação do modelo de análise, foi selecionada uma das cinco grandes áreas da cidade de Campinas, que podem ser vistas na figura 7, a área escolhida foi a área 1, em roxo. 
Tabela 8: Total de residentes por UTB na área 1 da Região Metropolitana de Campinas

\begin{tabular}{|c|c|c|c|c|c|c|}
\hline UTB & BAIRROS & Área $\left(\mathrm{Km}^{2}\right)$ & $\begin{array}{l}\text { População } \\
\text { Residente }\end{array}$ & $\begin{array}{c}\text { Domicílios } \\
\text { Permanentes }\end{array}$ & $\begin{array}{c}\text { Hab/ } \\
\text { Domicílios }\end{array}$ & $\mathrm{hab} / \mathrm{Km}^{2}$ \\
\hline 45 & Pq. Valença & 14,44 & 50334 & 14447 & 3,48 & 3485,73 \\
\hline $45 A$ & Residencial São Luiz & 0,184 & 2381 & 584 & 4,08 & 12908,83 \\
\hline 48 & $\begin{array}{l}\text { Mauro Marcondes, } \\
\text { Ouro Verde, Vista } \\
\text { Alegre }\end{array}$ & 14,624 & 62313 & 18255 & 3,41 & 4261,13 \\
\hline 49 & Maria Rosa & 4,254 & 9542 & 2885 & 3,31 & 2243,12 \\
\hline 50 & $\begin{array}{l}\text { São Cristóvão, Jd. } \\
\text { Planalto }\end{array}$ & 3,605 & 18074 & 5122 & 3,53 & 5013,61 \\
\hline 51 & DICS COHAB & 4,449 & 39733 & 11788 & 3,37 & 8931,45 \\
\hline 52 & $\begin{array}{l}\text { Distrito Industrial de } \\
\text { Campinas e Nova } \\
\text { Mercedes }\end{array}$ & 5,687 & 6499 & 1826 & 3,56 & 1142,72 \\
\hline 54 & $\begin{array}{l}\text { Jd. Atlântico, Jd. } \\
\text { Columbia }\end{array}$ & 7,068 & 1777 & 490 & 3,63 & 251,43 \\
\hline 65 & Nova Mercedes & 5,336 & 7507 & 2392 & 3,14 & 1406,74 \\
\hline 66 & $\begin{array}{l}\text { Jd. São Domingos, Jd. } \\
\text { Campo Belo }\end{array}$ & 6,089 & 19013 & 5410 & 3,51 & 3122,49 \\
\hline $66 \mathrm{~A}$ & Jd. Nova América & 1,376 & 5956 & 1760 & 3,38 & 4329,65 \\
\hline 67 & $\begin{array}{l}\text { Jd. Fernanda, } \\
\text { Campituba, Jd. } \\
\text { Itaguaçú }\end{array}$ & 3,921 & 18588 & 5111 & 3,64 & 4740,48 \\
\hline
\end{tabular}

As áreas em negrito na tabela 8 correspondem aos pontos utilizados para a elaboração de um cenário de uma rota otimizada que, saisse do Aeroporto Viracopos com destino a plataforma satélite UTB 48 Mauro Marcondes e que a partir da plataforma satélite passaasse por todos os pontos, considerando que em todas as marcações houvessem demandas aleatórias. A melhor rota encontrada por meio da ferramenta google mapas do Aeroporto Viracopos até a plataforma satélite selecionada leva 23 minutos com 11,9km de distância.

\subsection{CENÁRIO - ÁREA 1}

A partir da seleção de cinco pontos de estudo dentro da área 1 da cidade de Campinas, que podem ser vistos na tabela 9, foi possível, a partir da ferramenta google mapas, obter as distâncias de um ponto à outro, como está descrito na tabela 10. 
Tabela 9: Áreas em estudo

\begin{tabular}{|c|c|}
\hline Área & Localidade \\
\hline 1 & Conjunto Mauro Marcondes \\
\hline 2 & Residencial São Luis \\
\hline 3 & Distrito Industrial de Campinas \\
\hline 4 & Nova Mercedes \\
\hline 5 & Jardim Fernanda \\
\hline
\end{tabular}

Tabela 10: Distância entre as áreas em km

\begin{tabular}{|c|c|c|c|c|c|}
\hline & $\mathbf{1}$ & $\mathbf{2}$ & $\mathbf{3}$ & $\mathbf{4}$ & $\mathbf{5}$ \\
\hline $\mathbf{1}$ & 0 & 10,2 & 8,5 & 10,6 & 19,6 \\
\hline $\mathbf{2}$ & 9,8 & 0 & 16,4 & 26,9 & 33,8 \\
\hline $\mathbf{3}$ & 9,7 & 21,3 & 0 & 6,2 & 7 \\
\hline $\mathbf{4}$ & 11,2 & 19 & 6,3 & 0 & 11,2 \\
\hline $\mathbf{5}$ & 16,8 & 29,7 & 7,8 & 8,1 & 0 \\
\hline \multicolumn{7}{|c|}{ FONTE: Google Mapas } \\
\hline
\end{tabular}

Utilizando o modelo de programação linear do problema do caixeiro viajante, visto anteriormente, foi possível obter a melhor rota que percorre $52,4 \mathrm{~km}$ e passa do conjunto Mauro Marcondes para o Distrito Industrial de Campinas, do Distrito Industrial de Campinas para o Jardim Fernanda, do Jardim Fernanda para o bairro Nova Mercedes, do bairro Nova Mercedes para o Residencial São Luis, e por fim, do Residencial São Luis retorna para o conjunto Mauro Marcondes.

\subsection{EMISSÃO DE POLUENTES NO CENTRO URBANO - CENÁRIO 1}

A rota ótima dada pelo Solver possui 52,4 quilometros, o que gera uma determinada emissão de poluentes pela queima de combustível. Nesse e nos próximos cenários, o combustível em questão será o diesel e diferentes misturas de biodiesel e diesel, já que os veículos utilizados para o transporte de em sua grande maioria utilizam esses combustíveis.

A partir das tabelas 5 e 6 , foi possível calculas a emissão de $\mathrm{CO} 2$ em quilogramas durante 0 percurso, o que pode ser visto na tabela abaixo.

Tabela 11:Emissão de CO2 no Percurso do Cenário 1

\begin{tabular}{|c|c|}
\hline Combustível & Emissões em kg de CO2 no percurso \\
\hline Diesel & 67,072 \\
\hline Biodiesel B5 & 64,5568 \\
\hline Biodiesel B10 & 62,0416 \\
\hline Biodiesel B20 & 57,0112 \\
\hline Biodiesel B100 & 14,75584 \\
\hline
\end{tabular}




\subsection{CARREGAMENTO DO VEÍCULO NO AEROPORTO - CENÁRIO 1}

Para o transporte de carga do Aeroporto Viracopos até a plataforma satélite localizada no conjunto Mauro Marcondes, foi considerada a utilização de um veículo urbano de carga (VUC), que é um caminhão de menor porte, mais apropriado para áreas urbanas. Tal veículo apresenta comprimento máximo de 6,3 metros, largura máxima de 2,2 metros e capacidade de carga de 3 toneladas( COELHO, 2010).

Considerando que nem sempre o caminhão sai completamente cheio para as entregas e que além da variável peso ainda existem outras variáveis como o volume, foi considerada que dentro da capacidade de 3 toneladas do veículo, apenas 2 toneladas seriam utilizadas.

Foi considerado também que o carregamento do veículo seria feito por compras, do mesmo cliente ou de clientes que residem próximo, a fim de se estar na mesma rua o menor número de vezes possíveis dentro da semana. O critério utilizado para definir a urgência da entrega, foi de acordo com os dias restantes de prazo para a entrega, que foram estabelecidos para cada compra de forma aleatória, utilizando a função PROCV na tabela 12 para se encontrar o número aleatório gerado na tabela 13 e assim se obter a urgência da entrega, como está descrito nas tabelas abaixo.

Tabela 12: Prazos e Urgência para entrega das Compras

\begin{tabular}{|c|c|c|c|c|}
\hline Prazos & Urgência & Inicio & Fim & Urgência \\
\hline 1 & 10 & 0 & 0,1 & 10 \\
\hline 2 & 9 & 0,1 & 0,2 & 9 \\
\hline 3 & 8 & 0,2 & 0,3 & 8 \\
\hline 4 & 7 & 0,3 & 0,4 & 7 \\
\hline 5 & 6 & 0,4 & 0,5 & 6 \\
\hline 6 & 5 & 0,5 & 0,6 & 5 \\
\hline 7 & 4 & 0,6 & 0,7 & 4 \\
\hline 8 & 3 & 0,7 & 0,8 & 3 \\
\hline 9 & 2 & 0,8 & 0,9 & 2 \\
\hline 10 & 1 & 0,9 & 1 & 1 \\
\hline
\end{tabular}


Tabela 13: Composição das Compras, Peso e Urgência

\begin{tabular}{|c|c|c|c|c|}
\hline Compra & Conteúdo & Peso (kg) & Aleatório & Urgência \\
\hline 1 & Geladeira, Tv e fogão & 230 & 0,215895 & 8 \\
\hline 2 & Guarda-Roupa, maquina de lavar roupa e 2 livros & 262 & 0,049736 & 10 \\
\hline 3 & Cama e geladeira & 250 & 0,003092 & 10 \\
\hline 4 & Som, roupas, guarda-roupa & 213 & 0,841278 & 2 \\
\hline 5 & Celular, cama e maquina de lavar roupa & 161 & 0,666277 & 4 \\
\hline 6 & Cama e geladeira & 250 & 0,854371 & 2 \\
\hline 7 & 3 roupas, televisão e fogão & 89 & 0,801447 & 2 \\
\hline 8 & Geladeira, guarda roupa & 350 & 0,10286 & 9 \\
\hline 9 & Guarda roupa, cama e 1 celular & 301 & 0,068128 & 10 \\
\hline 10 & Som, celular, Fogão, guarda roupa & 251 & 0,526548 & \multicolumn{2}{|c}{5} \\
\hline
\end{tabular}

Utilizando o Solver para a otimização do problema, a fim de maximizar o carregamento de compras mais urgentes sem ultrapassar o limite de carga, temos que ideal é carregar as compras $1,2,3,5,7,8,9$ e 10 .

\subsection{O PROBLEMA DE ENTREGA LOCAL}

O cenário 1 apresenta uma configuração genérica, onde apenas um veículo atende a demanda de uma área muito grande, a fim de tornar o cenário mais favorável, foram elaborados os próximos cenários. Os cenários seguintes foram elaborados considerando que o veículo (VUC) que percorreu o caminho do Aeroporto Viracopos até a plataforma satélite no conjunto Mauro Marcondes, ao chegar à plataforma tem sua carga dividida em outros três veículos menores, de capacidade de carga reduzida, e que esses três veículos são enviados para cobrir cada um uma sub áreas descritas na figura 8 abaixo. 
Figura 8: Área 1 da cidade de Campinas subdividida

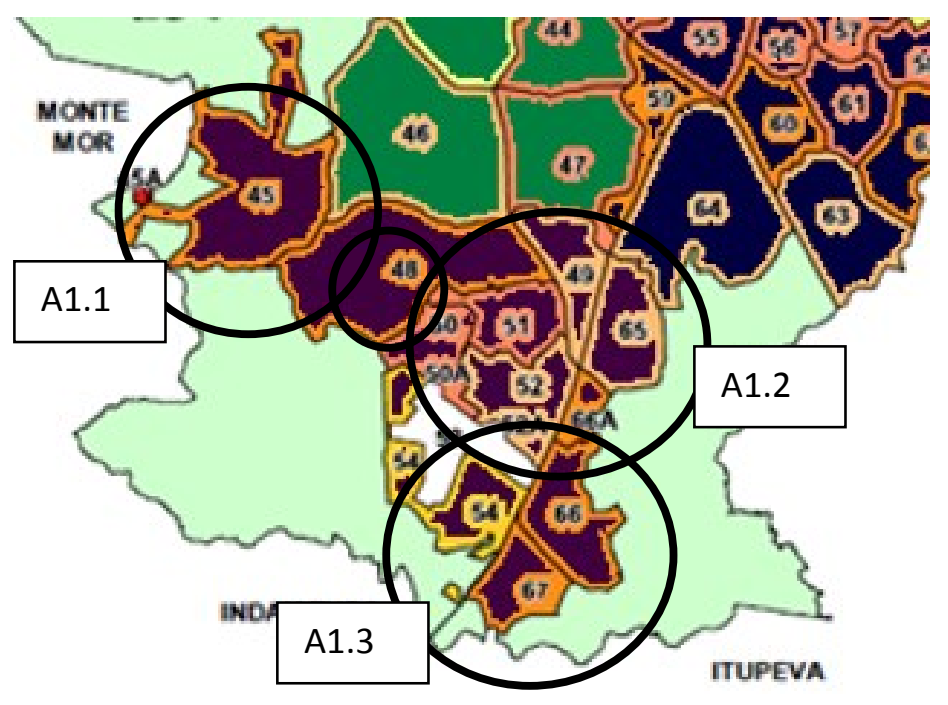

FONTE: (Plano Diretor de Campinas, 2010)

\subsection{ENTREGAS NA SUBÁREA A1.1}

O cenário 2 compreende a aplicação do problema do caixeiro viajante em pontos da área A1.1, identificada na figura 8 , e composta pelas UTBs presentes na tabela 14

Tabela 14: Total de residentes por UTB na subárea 1 da área 1 de Campinas

\begin{tabular}{|c|c|c|c|c|c|c|c|}
\hline UTB & BAIRROS & $\begin{array}{l}\text { Área } \\
\left(\mathrm{Km}^{2}\right)\end{array}$ & $\begin{array}{l}\text { População } \\
\text { Residente }\end{array}$ & $\begin{array}{c}\text { Domicílios } \\
\text { Particulares } \\
\text { Permanentes }\end{array}$ & Hab/Domicílios & $\mathrm{hab} / \mathrm{Km}^{2}$ & hab/hectare \\
\hline 45 & Pq. Valença & 14,44 & 50334 & 14447 & 3,48 & 3485,73 & 34,9 \\
\hline $45 A$ & $\begin{array}{l}\text { Residencial São } \\
\text { Luiz }\end{array}$ & 0,184 & 2381 & 584 & 4,08 & 12908,83 & 129,1 \\
\hline
\end{tabular}

FONTE: (Plano Diretor de Campinas, 2010)

A partir da seleção de quatro pontos de estudo dentro da subárea1 da área 1 da cidade de Campinas mais a plataforma satélite localizada no conjunto Mauro Marcondes, que podem ser vistos na tabela 15 , foi possível, a partir da ferramenta google mapas, obter as distâncias de um ponto à outro, como está descrito na tabela 16 .

Tabela 15: Áreas em estudo

\begin{tabular}{|c|c|}
\hline Área & Localidade \\
\hline 1 & Conjunto Mauro Marcondes \\
\hline 2 & Residencial São Luis \\
\hline 3 & Pq. Valença \\
\hline 4 & Jardim Rossin \\
\hline 5 & Av. dos Inajás \\
\hline
\end{tabular}


Tabela 16: Distância entre as áreas em km

\begin{tabular}{|c|c|c|c|c|c|}
\hline & $\mathbf{1}$ & $\mathbf{2}$ & $\mathbf{3}$ & $\mathbf{4}$ & $\mathbf{5}$ \\
\hline $\mathbf{1}$ & 0 & 10,2 & 8,4 & 6,9 & 10,7 \\
\hline $\mathbf{2}$ & 9,8 & 0 & 2,7 & 5,8 & 3,6 \\
\hline $\mathbf{3}$ & 8 & 2,7 & 0 & 4,4 & 3,8 \\
\hline $\mathbf{4}$ & 9,2 & 5,8 & 4,4 & 0 & 6,5 \\
\hline $\mathbf{5}$ & 10,3 & 3,6 & 3,8 & 6,6 & 0 \\
\hline
\end{tabular}

FONTE: Google Mapas

Utilizando o modelo de programação linear do problema do caixeiro viajante, visto anteriormente, foi possível obter a melhor rota que percorre $34,1 \mathrm{~km}$ e passa do conjunto Mauro Marcondes para o Residencial São Luis, do Residencial São Luis para o Parque Valença, do Parque Valença para o Jardim Rossin, do Jardim Rossin para Avenida dos Inajás e por fim, da Avenida dos Inajás retorna para o conjunto Mauro Marcondes.

\section{CONCLUSÃO}

Esse modelo pode ser utilizado em outros cenários de transporte de mercadorias em cidades, como nas outras áreas e subáreas de Campinas, porém não é possível chegar a uma solução ou modelo que solucione ou minimize todos os problemas do transporte de carga em meio urbano, já que os variados locais, veículos, mercadorias, entre outras variáveis, fazem com que cada situação seja única.

\section{REFERÊNCIAS}

EMBERGER, G. Urban Freight Transport Measures. City of Tomorrow, PLUMA -Planning of Mobility in Europe. 2004.

AWASTHI, A.; PROTH, J. M.A Systems- Based Approach for City Logistics Decision Making. JournalofAdvances in Management Research. 2006.

Revista Biotecnologia Ciência \& Desenvolvimento - Edição no 31 - julho/dezembro 2003. Disponível em:< http://www.geocities.ws/bueno_reis/biodiesel.pdf> Acesso em 10 de Setembro de 2014.

CARVALHO, C. H. R. Emissões relativas de poluentes do transporte motorizado de passageiros nos grandes centros urbanos brasileiros. IPEA: instituto de pesquisa econômica aplicada. Brasília, abril de 2011.

17ำ Congresso Brasileiro de Transporte e Trânsito, João Ernesto Toppan Nogueira Marcelo A. Darbello, Curitiba, 30 de setembro de 2009 (NOGUEIRA, J., DARBELLO, M.,2009)

ELKINGTON, J.:(1998) Partnerships from Cannibals with Forks: The Triple Bottom line of 21st Century Business, Environmental Quality Management, Stony Creek, CT: New Society Publishers 37-51.

BALOU, R. H.: (2001) Gerenciamento da cadeia de suprimentos, 4a. Edição, Bookman, Porto Alegre.

BELFIORE, Patrícia; FÁVERO, Luiz Paulo. Pesquisa Operacional para cursos de Engenharia. Elsevier Brasil, 2013.

MOORE, J.\& WEATHERFORD, L. (2006). Tomada de decisões em administração com planilhas eletrônicas. 6ạ edição.. 
LACHTTERMACHER, G. (2009) Pesquisa Operacional na Tomada de Decisão, 4a . Edição, Editora Pearson, São Paulo.

DABLANC, L., 2007Goods transport in large European cities: difficult to organize, difficult to modernize.Transportation Research Part A, vol. 41, p. 280-285.)

CRAINIC, T. G., RICCIARDI, N., STORCHI, G. Advanced freight transportation systems for congested urban areas, 2004.

QUAK, H. (2008) Sustainability of urban freight transport retail distribution and local regulations in cities. Erasmus Research Institute of Management (ERIM), Erasmus University Rotterdam, Ph.D. Thesis.)

COELHO, C. L., Logística descomplicada. 28 de Novembro de 2010.Disponível em: <http://www. logisticadescomplicada.com/tipos-de-caminhoes-tamanhos-e-capacidades/ >. Acessoem 20 de Abril de 2016.

BENJELLOUN, A., CRAINIC, T.G. Trends, challenges, and perspectives in city logistics.BuletinulAGIR, Simulating the impact of new Australian "bi-modal" urban freight terminals, 2009

Governo de Campinas. Disponível em: <http://www.campinas.sp.gov.br/governo/seplama/publicacoes/censo2010.php>. Acesso em 26 de Novembro de 2015.

Maciel, L. Falta Debate nas decisões sobre transporte de carga, Logistica Urbana, VALOR, 31/07/2013. 